\title{
Original
}

\section{Caracterización de mujeres en trabajo de parto a las que se les aplicó Maniobra Kristeller}

\section{Characterization of women in labor to whom the Kristeller Maneuver was applied \\ Carlos Alberto Ochoa Fletes ${ }^{1}$, Cesar Augusto Banegas Ávila², Yessica Turcios Hernández ${ }^{3}$, Marvin Enrique Rodríguez Moncada ${ }^{4}$, Roger Edilberto Linares Mendoza ${ }^{5}$}

${ }^{1}$ Servicio de Maternidad, Hospital San Felipe, Tegucigalpa, Honduras.

2,3,4,5 Universidad Católica de Honduras, campus Tegucigalpa.

*Autor para correspondencia: caofgo@gmail.com

\section{Palabras clave}

Efectos obstétricos adversos, violencia contra la mujer, violencia obstétrica, maniobra Kristeller.

\section{Keywords}

Obstetric side effects, violence against women, obstetric violence Kristeller maneuver.

\section{Historia del artículo}

Recepción: 01 agosto 2020

Aprobación: 25 mayo 2021

DOI: $\quad$ https://doi.org/10.5377/ rcfh.v7i1.11640

Citar como: Ochoa-Fletes CA, Banegas Ávila CA, Turcios Hernández $\mathrm{Y}$, Rodríguez Moncada ME, LinaresMendoza RE. Caracterización de mujeres en trabajo de parto a las que se les aplicó maniobra Kristeller. Rev. cienc. forenses Honduras. 2021; 7(1): 15-22. DOI: https://doi.org/10.5377/ rcfh.v7i1.11640

Declaración de relaciones y actividades financieras y Declaración de conflictos de interés: ninguna

\section{RESUMEN}

Introducción: en la obstetricia se realizan algunas prácticas que producen daños significativos a la madre o a su bebé, aunque no siempre se admite su ejecución. Un ejemplo es la maniobra de Kristeller, cuya aplicación es controvertida. Objetivo: determinar la frecuencia con que se realizó la maniobra de Kristeller en una muestra de mujeres atendidas en la maternidad del Hospital San Felipe de Tegucigalpa. Metodología: estudio descriptivo, que se realizó en el período comprendido entre los meses de marzo a mayo del 2018. Se incluyeron un total de 376 pacientes. Las variables estudiadas fueron: hora del parto, paridad, profesional que atendió y supervisó el parto, profesional que realizó la maniobra de Kristeller, complicaciones maternas y neonatales observadas. Resultados: se encontró una frecuencia de maniobra de Kristeller de $12.2 \%$ (46/376). Conclusiones: ser primigesta incrementó el riesgo de que se le aplicara la maniobra Kristeller (OR: 3.11, IC:95\%, $p=0.0002$ ), dentro de los factores protectores se encontraron ser multípara (OR:0.25, IC:95\%, $p=0.00004)$ y, haber sido atendida por una licenciada en enfermería (OR: 0.34, IC:95\%, $p=0.0097)$. La maniobra de Kristeller aumentó el riesgo de complicaciones maternas en 12.5 veces (OR: 17.6, IC:95\%, $p=0.0000000019$ ) y 6.5 veces en el neonato (OR: 7.7 IC:95\%, $p=0.0000578)$. 


\section{ABSTRACT}

Introduction: in obstetrics, there are some practices carried out that can cause damage to the mother or her baby, although their execution is not always allowed. An example is the Kristeller's maneuver, and its application is controversial. Objective: to determine the frequency with which the Kristeller maneuver was performed in a sample of women cared for in the maternity ward of the San Felipe General Hospital of Tegucigalpa. Methodology: descriptive study, carried out in the period from March to May 2018. A total of 376 patients were included. The variables studied were time of birth, parity, professional who attended and supervised the delivery, professional who performed the Kristeller maneuver, maternal and neonatal complications observed. Results: a Kristeller maneuver frequency of $12.2 \%$ (46/376) was found. Conclusions: being pregnant for the first time increases the risk of resorting to the Kristeller maneuver (OR: 3.11, $\mathrm{Cl}: 95 \%, p=0.0002775)$, within the protective factors are, being multiparous (OR:0.25, Cl: $95 \%, p=0.0000418$ ) and, to have been attended by a nursing professional (OR: $0.34, \mathrm{Cl}: 95 \%$, $p=0.0097920)$. Kristeller's maneuver increases the risk of maternal complications by 12.5 times (OR: 17.6, Cl: 95\%, $p=0.0000000019$ ) and 6.5 times in the neonate (OR: $7.7 \mathrm{Cl}: 95 \%$, $p=0.0000578)$.

\section{INTRODUCCION}

En la práctica de la obstetricia frecuentemente se realizan procedimientos que podrían tener repercusiones negativas en la madre y/o en el bebé; entre ellas podemos mencionar la maniobra de Kristeller, que fue descrita en 1867 por Samuel Kristeller y consiste en hacer presión sobre el fondo uterino durante el período expulsivo ${ }^{1}$. Se ha publicado información controvertida al respecto de su utilidad; en una revisión sistemática, los autores reportaron que: "No se encontró evidencia suficiente para establecer conclusiones sobre los efectos beneficiosos o perjudiciales de la presión del fondo uterino, ya sea manualmente o mediante un cinturón inflable", sin embargo otros autores concluyeron que la maniobra de Kristeller no incrementa la tasa de partos vaginales espontáneos, ni reduce la tasa de parto instrumental 2,3. Otros estudios reportaron que ocasiona diversos daños en la madre, siendo los más frecuentemente descritos dolor, ruptura uterina, desgarros vaginales y perineales, hemorragia postparto, fractura de costillas, inversión uterina, desprendimiento de placenta, muerte materna entre otras 4,5. Entre las complicaciones neonatales se han descrito fracturas, principalmente de clavícula, parálisis de Erb, hemorragia intraocular, taquipnea transitoria, asfixia neonatal y cefalohematomas ${ }^{6}$. Pese a
Rev. cienc. forenses Honduras, Volumen 7, N 1, Año 2021

Open Access $\quad$ CAutor(es).
URL: http://www.bvs.hn/RCFH/html5/

CC-BY-NC 4.0 internacional 
que la Organización Mundial de la Salud desaconseja su uso ${ }^{7}$, aún se usa frecuentemente ${ }^{8-11}$.

En Honduras, en el año 2016, por primera vez aparece en las normas nacionales la figura de "violencia obstétrica", establecida como un mecanismo para la protección de la madre y su bebé de prácticas dañinas ${ }^{12}$. A partir de esta normativa la realización de ciertas prácticas, incluyendo la maniobra de Kristeller, son consideradas como fallas en los estándares de atención y por ende objeto de imputación por fallas en la responsabilidad profesional, se estima que esto podría ser un factor que favorece el subregistro, lo que a su vez dificulta cuantificar el daño producido ${ }^{13}$.

Por lo anteriormente expuesto se considera que conocer la frecuencia con que se utiliza, así como los posibles efectos adversos asociados a su uso, es de suma importancia. Esta investigación tuvo por objetivo describir las características y frecuencia con que se realizó la maniobra de Kristeller, en la Maternidad del Hospital de Especialidades San Felipe de Tegucigalpa, Honduras.

\section{METODOLOGÍA}

Se realizó un estudio descriptivo, retrospectivo. La selección de las participantes se hizo al azar.
Se entrevistó a las pacientes puérperas ingresadas en las salas de puerperio 1 y 2 ; seis pacientes por sala, durante el periodo comprendido entre el 01 de marzo al 31 de mayo de 2018, para un total de 376 pacientes incluidas.

Para facilitar la comprensión de las pacientes se les mostraron cartillas con imágenes representando varios tipos de maniobras de Kristeller. A todas las pacientes se les explicó el objetivo del estudio $y$ todas firmaron un consentimiento informado autorizando incluir su información en este estudio. Se revisó el expediente clínico de las pacientes incluidas, las variables estudiadas fueron: hora en la que se produjo el parto, paridad, profesional que atendió y supervisó el parto, profesional que realizó la maniobra de Kristeller, complicaciones maternas y neonatales presentadas.

El análisis de los datos se realizó con el programa Epi Info $7.2 .2 .2^{\circledR}$, se calculó el Odd ratio $(O R)$ entre las pacientes que fueron sometidas a la maniobra y las que no lo fueron, con un intervalo de confianza de 95\% (IC 95\%) y un valor $p=0.05$.

\section{RESULTADOS}

El $34.7 \%(16 / 46)$ de los partos estudiados 
asociados con maniobra de Kristeller se dieron entre las 22:00 a 01:00 horas y se encontró una frecuencia de la maniobra de Kristeller de 12.2 $\%$ (46/376); se aplicó más frecuentemente en las primigestas (32/46) que en las multíparas (14/46). El $50.4 \%$ de los partos fueron atendidos por médicos internos, sin embargo, se encontró que la aplicación de la maniobra fue menos frecuente en los partos atendidos por licenciadas en enfermería con un 5.5\% (5/86) versus $13.7 \%(26 / 190)$ de los partos atendidos por médicos internos.

El $47.2 \%$ de los partos fue supervisado ya sea por un médico especialista, un médico general o una licenciada en enfermería, sin embargo, la supervisión no representó un valor agregado, al contrario, la maniobra se realizó con menor frecuencia en los partos no supervisados $4.5 \%$ (9/199) versus $21.6 \%$ (37/171) de los partos supervisados.

Se observaron complicaciones en el $30.4 \%$ de las puérperas a las que se les realizó la maniobra de Kristeller y en el $19.6 \%$ de sus recién nacidos. De la población estudiada 330 pacientes no fueron sometidas a la maniobra de Kristeller durante el expulsivo, en las cuales se observó complicaciones maternas únicamente en el $2,4 \%$ y $3 \%$ de complicaciones neonatales, tal y como se muestra en los cuadros $\mathbf{1}$ y 2.

CUADRO №1. Frecuencia de las complicaciones maternas en puérperas a las que se les realizó la maniobra de Kristeller versus las no sometidas a la maniobra.

\begin{tabular}{|c|c|c|c|c|c|c|}
\hline \multirow{2}{*}{$\begin{array}{l}\text { Complicación } \\
\text { materna }\end{array}$} & \multicolumn{2}{|c|}{$\begin{array}{c}\text { Con Kristeller } \\
n=46\end{array}$} & \multicolumn{2}{|c|}{$\begin{array}{c}\text { Sin Kristeller } \\
n=330\end{array}$} & \multirow{2}{*}{$\begin{array}{c}\text { OR } \\
95 \% \text { IC }\end{array}$} & \multirow[t]{2}{*}{ Valor $p$} \\
\hline & Frecuencia & $\%$ & Frecuencia & $\%$ & & \\
\hline Desgarro & 10 & 18.1 & 5 & $1.3 \%$ & $\begin{array}{c}18.1 \\
5.9-55.9\end{array}$ & 0.001 \\
\hline $\begin{array}{l}\text { Hemorragia post } \\
\text { parto }\end{array}$ & 2 & 7.5 & 2 & $0.5 \%$ & $\begin{array}{c}7.4 \\
1.0-54.4\end{array}$ & 0.02 \\
\hline Retención de placenta & 1 & 7.1 & 1 & $0.26 \%$ & $\begin{array}{c}7.3 \\
0.4-119.3\end{array}$ & 0.1 \\
\hline
\end{tabular}

Fuente: Expedientes clínicos, Servicio de Maternidad Hospital San Felipe, 2018.

Rev. cienc. forenses Honduras, Volumen 7, N 1, Año 2021

Open Access CAutor(es).
URL: http://www.bvs.hn/RCFH/html5/ CC-BY-NC 4.0 internacional 


\section{CUADRO №2. Frecuencia de complicaciones neonatales en partos donde se aplicó maniobra}

de Kristeller versus las no sometidas a la maniobra.

\begin{tabular}{|c|c|c|c|c|c|c|}
\hline \multirow{2}{*}{$\begin{array}{c}\text { Complicación } \\
\text { materna }\end{array}$} & \multicolumn{2}{|c|}{$\begin{array}{c}\text { Con Kristeller } \\
n=46\end{array}$} & \multicolumn{2}{|c|}{$\begin{array}{c}\text { Sin Kristeller } \\
n=330\end{array}$} & \multirow{2}{*}{$\begin{array}{c}\text { OR } \\
95 \% \text { IC }\end{array}$} & \multirow{2}{*}{$\begin{array}{c}\text { Valor } \\
\mathbf{p}\end{array}$} \\
\hline & Frecuencia & $\%$ & Frecuencia & $\%$ & & \\
\hline CAPUT & 3 & 3.8 & 6 & $1.6 \%$ & $\begin{array}{c}3.8 \\
0.9-15.7\end{array}$ & 0.05 \\
\hline $\begin{array}{l}\text { Depresión } \\
\text { respiratoria }\end{array}$ & 5 & 13.3 & 3 & $0.8 \%$ & $\begin{array}{c}13.3 \\
3.1-57.8\end{array}$ & $<0.001$ \\
\hline $\begin{array}{l}\text { Ingreso } \\
\text { hospitalario }\end{array}$ & 1 & 7.3 & 1 & $0.27 \%$ & $\begin{array}{c}7.3 \\
0.4-119.3\end{array}$ & 0.1 \\
\hline
\end{tabular}

Fuente: Expedientes clínicos, Servicio de Maternidad Hospital San Felipe, 2018.

\section{DISCUSIÓN}

La maniobra de Kristeller es un procedimiento obstétrico controvertido, la OMS la considera dentro la categoría C: "debería de usarse con cautela, hasta que nuevos estudios clarifiquen el tema" ${ }^{14}$, pese a esto se estima que se realiza entre el $25 \%$ y el $89 \%$ de los partos ${ }^{8-11,15}$. Nuestro estudio encontró una frecuencia del 12\%; se practicó en uno de cada 10 partos. Se estima que esta maniobra se realiza en la mayoría de los hospitales del mundo, pero la frecuencia de su aplicación esta subregistrada en el expediente clínico ${ }^{13}$, posiblemente por el temor de ser objeto de una demanda por responsabilidad profesional, ya que hay evidencia que indica su relación con lesiones maternas y neonatales. En un trabajo realizado en Arequipa, Perú, publicado el 2017, donde estudiaron los hallazgos anatomo-patológicos forenses y su diagnóstico perinatal de asfixia, donde encontraron 35 casos de asfixia perinatal de un total de 2680 necropsias, dentro de las cuales, una de las causas más importantes presentada fue el trauma obstétrico con un $31.43 \%$, que podría estar asociado a la maniobra de Kristeller $^{15}$. Se reportó además que la maniobra se realizó con mayor frecuencia en primigestas en un $69.5 \%$, el horario donde más se practicó fue entre las 22:00hrs-01:00hrs, en un $34.8 \%$ de los casos, siendo ambos factores de riesgo. Además, se observaron complicaciones en el $30.4 \%$ de las puérperas a las que se les realizó la maniobra de Kristeller, siendo las más frecuentes los desgarros vaginales (grado III y grado IV) (OR: 18.11, IC: 95\%, $p=0.001$ ) y la hemorragia postparto (OR: 7.4, IC: 95\%, $p=$ 
0.04), que incrementa su riesgo en 14.39 y 7.1 veces respectivamente ${ }^{15}$. En nuestro estudio encontramos que en el $21.7 \%$ de las pacientes a las que se les había realizado esta maniobra presentaron desgarros vaginales (grado III, grado IV), en consonancia a lo reportado por Peña y Heladia, donde observaron la realización de maniobras externas para la extracción del recién nacido (maniobra de Kristeller) en un $32 \%^{16}$.

El 19.6\% de los recién nacidos a cuyas madres se les había practicado la maniobra, presentó complicaciones, siendo las más frecuentemente observadas la depresión respiratoria (5 casos; $10.9 \%$ ), seguido del CAPUT ( 3 casos; $6.5 \%$ ), y por último la necesidad de ingreso en una sala de observación de recién nacidos (1 caso; 2.2\%), Peña y Heladia en México consideraron que esta maniobra no debería realizarse por los graves riesgos de que ocurran lesiones como fracturas, hemorragia intracraneal o cefalohematoma ${ }^{16}$.

Es importante destacar algunos hallazgos como el hecho que la maniobra se aplica con menor frecuencia cuando la atención del parto la realiza profesionales de enfermería, siendo los médicos titulados quienes realizaron la maniobra en 44 casos (95.6\%), médicos internos en un caso (2.2\%) versus un caso por personal de enfermería (2.2\%); esto podría estar condicionado por factores formativos, que inician en la etapa de estudiantes y termina convirtiéndose en una práctica habitual, entre los estudiantes de medicina, con la idea errónea de ser una práctica inocua.

\section{CONCLUSIONES}

La maniobra de Kristeller no es inocua y aunque su uso está proscrito por estar relacionada con complicaciones materno-perinatales., en la muestra analizada se encontró que aumenta la probabilidad de complicaciones maternas en 12.9 veces, e incrementa el riesgo de complicaciones neonatales en 6.5 veces; pese a que se desaconseja su uso, aún se aplica frecuentemente.

Se recomienda desarrollar programas de capacitación al personal de salud, hacer conciencia con evidencia de los peligros que involucra y resaltar la responsabilidad legal que puede derivar de su realización.

A pesar del subregistro o invisibilidad de la maniobra y dado el estándar de atención aprobado en la Secretaría de Salud hondureña, es recomendable realizar estudios de intervención con procesos de formación y generar la misma experiencia en otros hospitales del país para hacer comparaciones e intervenciones oportunas a fin de evitar las 
repercusiones en las pacientes y sus hijos, así como las consecuencias legales derivadas.

\section{Limitantes del estudio}

Debido a las implicaciones negativas de la maniobra, se considera una maniobra invisible (pero no inexistente), ya que esta tiende a ocultarse; recabar la información de manera retrospectiva de los expedientes implicó una limitante, porque usualmente no queda registrada; la frecuencia encontrada en este estudio no necesariamente refleja la realidad de ocurrencia.

\section{REFERENCIAS BIBLIOGRÁFICAS}

1.- Cunningham FG, Leveno K. J, Bloom SL, Spong CY, Dashe JS, Hoffman BL, Sheffield JS. Obstetricia de Williams. Brasil: McGraw Hill; 2016.

2.-Muñoz Martínez, AL, Berral Gutiérrez, MA; Burgos Sánchez JA. Es la presión en el fondo uterino durante el expulsivo (Kristeller) una maniobra a abandonar. Evidentia. [Internet]. 2009[citado 14 enero 2021];6(28). Disponible en: http://matronasubeda.objectis.net/areacientifica/produccion-cientifica-

propia/articulos/es-la-presion-en-el-fondo-

uterino-durante-el-expulsivo-kristeller-unamaniobra-a-abandonar

3.-Hofmeyr GJ, Vogel JP, Cuthbert A, Singata M. Fundal pressure during the second stage of labour. Cochrane Database of Systematic Reviews 2017, Issue 3. Art. No.: CD006067. DOI: 10.1002/14651858.CD006067.pub3.

4.- Fescina R, Schwarcz R, Salas S, Duverges C, et al. Obstetricia, 7a ed. Buenos Aires: El Ateneo; 2007.

5.- Youssef A, Salsi G, Cataneo I, et al. Fundal pressure in second stage of labor (Kristeller maneuver) is associated with increased risk of levator ani muscle avulsion. Ultrasound Obstet Gynecol. 2019;53(1):95-100. doi:10.1002/uog.19085

6.- Hayata E, Nakata M, Takano M, Umemura N, Nagasaki S, Oji A, et al. Safety of uterine fundal pressure maneuver during second stage of labor in a tertiary perinatal medical center: A retrospective observational study. Taiwan J Obstet Gynecol. 2019; 58(3):375-379. doi:10.1016/j.tjog.2018.10.033

7.-World Health Organization. Regional Office for Europe. Hospital care for mothers and newborn babies: quality assessment and improvement tool. $2 \mathrm{a}$ ed Copenhagen, Dinamarca: WHO; 2014.

8.-Hasegawa J, Sekizawa A, Ishiwata I, Ikeda T, Kinoshita K. Uterine rupture after the uterine fundal pressure maneuver. I Perinat Med. 2015;43(6):785-788. doi:10.1515/.jpm-20140284

9.-Cuerva MJ, Tobias P, Espinosa JA, Bartha JL. Ultrasonido intraparto antes de la maniobra de 
Kristeller: un estudio observacional. Revista de medicina perinatal. 2015;43(2):171-175. DOI: 10.1515 / jpm-2014-0079.

10.-Becerra-Chauca N, Failoc-Rojas VE. Maniobra Kristeller, consecuencias físicas y éticas según sus protagonistas. Rev Cubana Obstet Ginecol [Internet]. 2019[citado 07 agosto 2020];45(2): e331. Disponible en: http://scielo.sld.cu/scielo.php?script=sci_arttex t\&pid=S0138-600X2019000200004\&lng=es

11.- Do Carmo M, Esteves A, Madeira R, Theme $M$, Bastos $M$, Nakamura $M$, et al. Intervenções obstétricas durante o trabalho de parto e parto em mulheres brasileiras de risco habitual. Cad. Saúde Pública. 2014;30 Sup: S1-S31.

12.-Secretaria de Salud (HN) Atención durante la preconcepción, el embarazo, el parto, el puerperio y del neonato. [Internet]. Tegucigalpa: Secretaría de Salud; 2015. [citado 07 agosto 2020]. Disponible en: http://www.salud.gob.hn/site/index.php/comp onent/edocman/normas-atencion-durante-lapreconcepcion-embarazo-parto-puerperio-y$\underline{\text { neonato }}$

13.- Malvasi A, Zaami S, Tinelli A, Trojano G, Montanari Vergallo G, Marinelli E. Kristeller maneuvers or fundal pressure and maternal/neonatal morbidity: obstetric and judicial literature review. J Matern Fetal Neonatal Med. 2019;32(15):2598-2607. doi: 10.1080/14767058.2018.1441278

14.-Organización Mundial de la Salud. Grupo técnico de trabajo de la OMS. Departamento de Investigación y Salud Reproductiva. Cuidados en el parto normal: una guía práctica. Ginebra: OMS; 1996.

15.- Zapata Ponze de León M. Hallazgos anatomopatológicos forenses y su correlación con el diagnostico perinatal de asfixia, Instituto de Medicina Legal y Ciencias Forenses, Arequipa 2014 - 2016 [Tesis en línea]. Perú: Universidad Católica de Santa María; 2017. Disponible en: http://tesis.ucsm.edu.pe/repositorio/handle/U

\section{CSM/6599}

16.- García HJ, Peña A. Incidencia de lesiones maternas causas por la maniobra de Kristeller. Rev Méd Inst Mexl Seguro Soc. 2004;42(1):2530.

17.- Lattus J, Paredes A, Junemann K, Martic A, Contreras P, Baeza N, et al. Espatulas de Thierry versus forceps de Kjelland. Rev Chil Obstet Ginecol. 2003;68 (6):477- 486. 